\title{
A Sectional Control Method to Decrease the Accumulated Survey Error of Tunnel Installation Control Network
}

\author{
Ying-gang Guo*, Zong-chun Li \\ School of Geospatial Information, Information Engineering University, Zhengzhou, China \\ Email address: \\ fariel_gyg@163.come (Ying-gang Guo), 13838092876@139.com (Zongchun Li) \\ ${ }^{*}$ Corresponding author
}

\section{To cite this article:}

Ying-gang Guo, Zong-chun Li. A Sectional Control Method to Decrease the Accumulated Survey Error of Tunnel Installation Control Network. American Journal of Modern Physics. Vol. 10, No. 1, 2021, pp. 7-15. doi: 10.11648/j.ajmp.20211001.12

Received: March 1, 2021; Accepted: March 12, 2021; Published: March 17, 2021

\begin{abstract}
The location reference for the precision installation of components of particle accelerator is provided by tunnel installation control network. The long and narrow control network has big accumulated error with increasing distance. In order to decrease the accumulated survey error of tunnel installation control network of particle accelerator, a sectional control method is proposed. Firstly, the accumulation rule of positional error with the length of control network is obtained by simulation calculation according to the shape of tunnel installation control network. Then, the RMS of horizontal positional precision of tunnel backbone control network is taken as the threshold. When the accumulated error is bigger than the threshold, tunnel installation control network should be divided into subsections reasonably. On each segment, the middle survey station is taken as the datum for independent adjustment calculation. Finally, by taking the backbone control points as faint datums, the weighted partial parameters adjustment is performed with the adjustment results of each segment and the coordinates of backbone control points. The subsections are jointed and unified into the global coordinate system in the adjustment process. An installation control network of linac with a length of $1.6 \mathrm{~km}$ is simulated. The RMS of positional deviation of the proposed method is $2.583 \mathrm{~mm}$, and the RMS of the difference of positional deviation between adjacent points reaches $0.035 \mathrm{~mm}$. Experimental results show that the proposed sectional control method can not only effectively decrease the accumulated survey error, but also guarantee the relative positional precision of installation control network. So it can be applied in the data processing of tunnel installation control network, especially for large particle accelerators.
\end{abstract}

Keywords: Alignment, Tunnel Installation Control Network, Accumulated Survey Error, Sectional Control Method, Datum

\section{Introduction}

Particle accelerator is a kind of large-scale scientific facility for high energy physics experiments [1-3]. In order to realize the smoothness of beam orbit and ensure that the particles travel along the designed orbit and bombard the targets accurately, the installation accuracy of the accelerator components needs to reach sub-millimeter level $[4,5]$. The tunnel installation control network of particle accelerator is the direct survey reference for components alignment. Research on the design, survey and data processing of the installation control network is of great significance for the realization of accelerator scientific objectives $[6,7]$.

At present, laser tracker is the main tool for the installation control network survey and components alignment of particle accelerators [8-12]. Particle accelerators are generally built underground in tunnel, which determines that the tunnel installation control network is long and narrow. In the control network, laser trackers are firstly set up with free-station method, then the adjacent stations are jointed and transformed into a unified coordinate system with common points [13, 14]. This survey and data processing method will lead to obvious accumulated positional error with the increase of the control network length. For the reason that all the lengths of the particle accelerators which have been built in China so far are not more than a few hundred meters, the error accumulation of their control network is insignificant and negligible. However, for large particle accelerators under construction, such as the High Energy Photon Source (HEPS) with a circumference of $1360 \mathrm{~m}$ and the Circular 
Electron Positron Collider (CEPC) with a circumference of $100 \mathrm{~km}$, the issue of error accumulation in installation control network has to be solved $[15,16]$. It is reported in the CEPC conceptual design reported that the accumulated error of CSNS tunnel control network with a length of $228 \mathrm{~m}$ reaches $\pm 3.3 \mathrm{~mm}$ [17]. According to this, the accumulated error in the $100 \mathrm{~km}$ tunnel of CEPC will reach $1428 \mathrm{~mm}$, which is very amazing. Dominique calculated the accumulated error of the tunnel installation control network of the Future Circular Collider by simulation. The accumulated error reached $8 \mathrm{~mm}$ at $5 \mathrm{~km}$ away and reached $12 \mathrm{~mm}$ at $10 \mathrm{~km}$ away [18]. On how to reduce error accumulation, scholars at home and abroad have carried out relevant research. Yang established a joint adjustment model based on leveling data and three-dimensional coordinate data [14]. In this model, the height direction is constrained by high-precision leveling data, which effectively weakens the accumulated error in the elevation direction. To solve the issue that the coordinate survey error of the traditional multiple stations survey accumulates constantly with the increase of number of survey stations, Zhou et al. proposed two data fusion methods of overall adjustment [19], the positional precision of which is higher and more stable than traditional method. Lü et al. employed the overall adjustment method to process the original observation and the common point coordinate observation, which improves the precision of adjusted coordinates of multiple stations survey [20]. In the process of processing the data of GPS control network, by ascribing proper weights to reference stations, the reference stations not only provided reference frame but also caused little local distortion [21].

Inspired by these references, in order to decrease the error accumulation of installation control network of particle accelerators, this paper puts forward a new idea. Firstly, by taking the RMS of horizontal positional precision of backbone control points as the threshold, the tunnel installation control network need to be reasonably divided into subsections. Then, the observation of survey stations on each segment are adjusted independently by taking the middle station as mandatory datum. Finally, the adjustment results on each segment are jointed and unified to the global coordinate system with weighted partial parameters adjustment by taking the backbone control points as faint datums.

\section{Adjustment of Classical Free Network}

When utilizing tunnel installation control network to guide the alignment of components, the coordinates of the control points have to be known. However, we can only get the relative horizontal direction, zenith angle and slope distance from the instrument center to the control points with the free-station method of laser trackers, which cannot directly provide the coordinates of a global coordinate system. How to affiliate these relative observations with the unified reference frame involves the definition of the global coordinate system and the selection of adjustment datum.
The global coordinate system refers to a specific unified coordinate system. The coordinates of control points in the global coordinate system can provide a global positional reference for the installation and alignment of the components, so as to ensure the positional relationship among the linac, transfer line, booster and collider ring. A datum is some known quantities in a control network. It appears as the coordinates, zenith angles, side lengths, azimuths, and so on. It provides the necessary known data for adjustment calculation to restrict the position, size and direction of the control network, so that the adjustment has a unique solution $[22,23]$. If a control network lacks datums, we can only obtain the internal relative shape of the control network by those relative observations, but cannot calculate the coordinate estimation, covariance matrix, and so on. The tunnel installation-control- network is a three-dimensional control network. The necessary known data for a three-dimensional control network includes 3 position parameters, 3 orientation parameters and 1 scale parameter. More than 3 known control points can also be taken as the datum to provide the necessary known information. There are two kinds of datum, the mandatory datum and the faint datum $[24,25]$. A mandatory datum means that the form of the datum remains unchanged before and after adjustment. A faint datum is composed of random data, which will be corrected after adjustment.

To analyze the error accumulation issue of tunnel installation control network of particle accelerator, the installation control network of a linac with a length of $1.6 \mathrm{~km}$ is simulated. It is assumed that the width of the tunnel is $6 \mathrm{~m}$, and control points are evenly distributed at the intervals of 8 $\mathrm{m}$. There are 4 control points on each transect, 2 on the ground and 2 on the two sides of the tunnel wall. Laser tracker stations are set up in the middle of every adjacent transect to observe the control points on adjacent 6 transects, and 200 survey stations are needed to observe the entire control network. The distributions of the survey stations and control points of the tunnel installation control network is shown in Figure 1 and Figure 2.

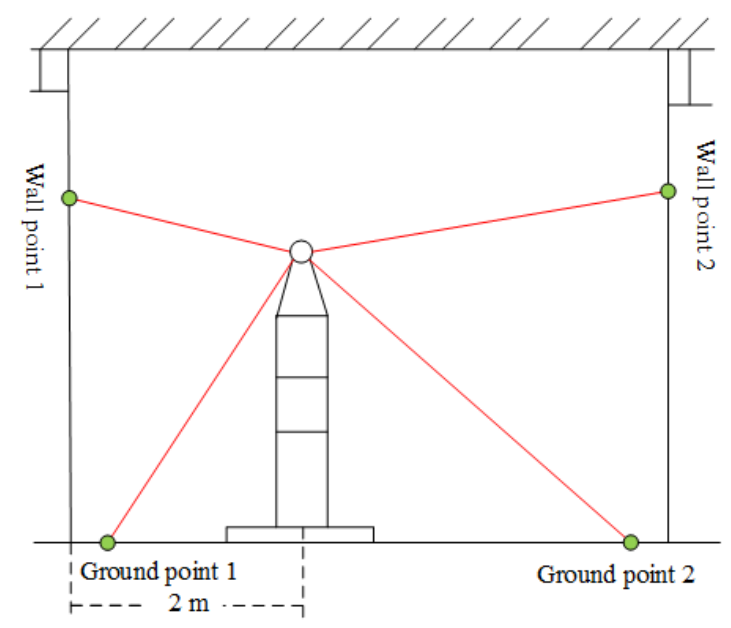

Figure 1. Distribution of control points on a transect. 


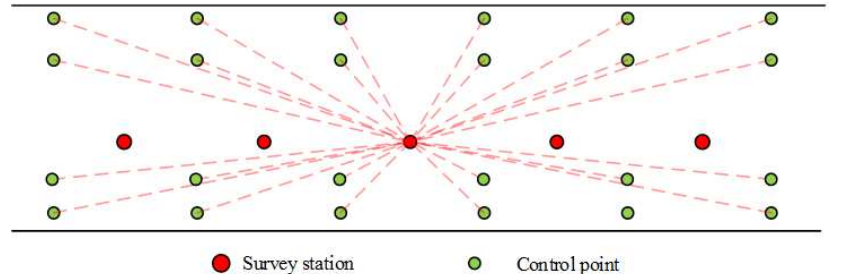

Figure 2. Distribution of survey stations and control points.

A classical free network is a control network which only contains the necessary datum data. If we take the instrument coordinate system of the first survey station as the global coordinate system, take the instrument center as the position datum, take the direction of the 3 coordinate axes as the orientation datum, and take the distance between the instrument center and a control point as the scale datum, this control network will be a classical free network with only necessary datum data. The coordinates of other survey stations and control points in the coordinate system of the first survey station are calculated by adjustment. The variation of positional mean square error with the distance from control points to the datum is shown in Figure 3, and the variation of the error ellipses is shown in Figure 4.

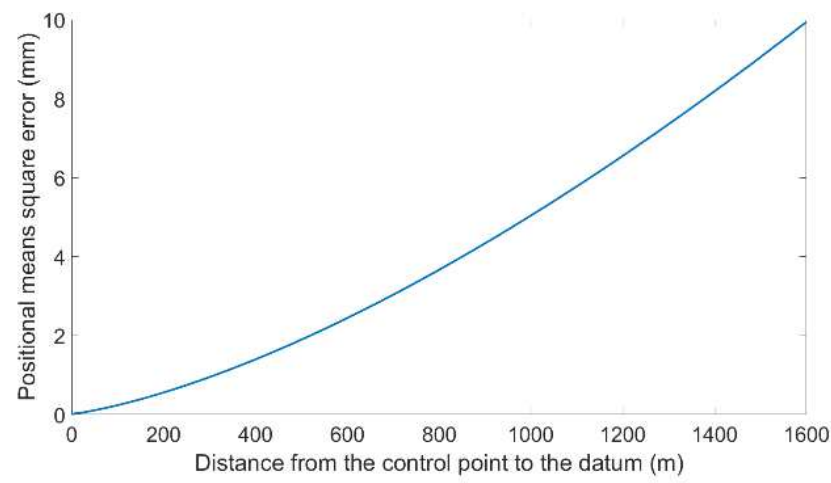

Figure 3. Variation of positional mean square error.

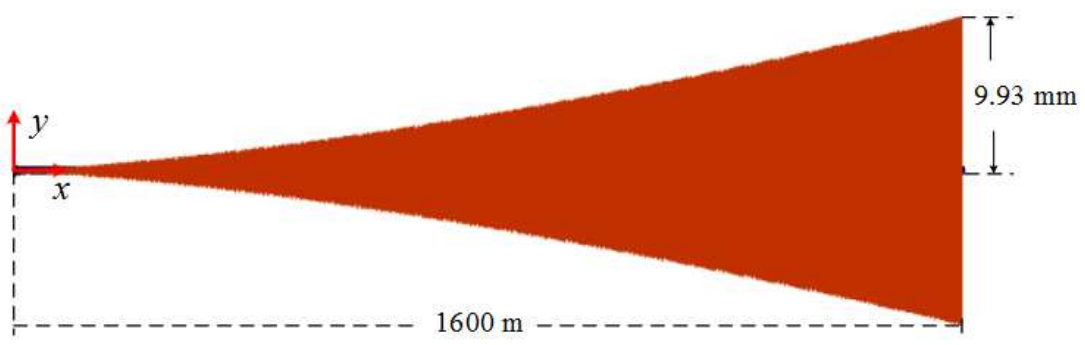

Figure 4. Variation of error ellipses.

Figure 3 and Figure 4 show that the positional mean square error and the size of error ellipses of control points increase with the distance from control point to the datum.

In order to evaluate the relative precision, the length of semi-major axis of relative error ellipses between two adjacent control points is calculated. The relative accuracy can be evaluated with the difference of deviation from the adjusted coordinate to the designed coordinates between two adjacent control points. Nine points are taken as an example at the intervals of $200 \mathrm{~m}$. The positional mean square error, the length of semi-major axis of error ellipses, the 3D deviation from the adjustment coordinates to the designed coordinates, and the length of semi-major axis of relative error ellipse of these points are listed in Table 1. The Precision statistical results of the adjustment result of classical free network are listed in Table 2. The relative error ellipses from these points to their adjacent points are shown in Figure 5. In Table 1, $m_{\mathrm{x}}$, $m_{\mathrm{y}}$ and $m_{\mathrm{z}}$ represent the mean square error in the $x, y$ and $z$ direction respectively, and $m_{P}=\sqrt{m_{x}^{2}+m_{y}^{2}+m_{z}^{2}}$ represents the $3 \mathrm{D}$ positional mean square error. $\mathrm{E}_{1}$ represents the length of semi-major axis of error ellipse, and $E_{2}$ represents the length of semi-major axis of relative error ellipse. In Table 2, $\mathrm{E}_{3}$ represents the Maximum of the difference of positional deviation between adjacent points, and $\mathrm{E}_{4}$ represents the RMS of the difference of positional deviation between adjacent points.

Table 1. Precision of some control points in classical free network.

\begin{tabular}{|c|c|c|c|c|c|c|c|}
\hline$/ \mathrm{mm}$ & & & & & & & \multirow{3}{*}{$\mathbf{E}_{2}$} \\
\hline \multirow{2}{*}{ Point name } & \multicolumn{4}{|c|}{ Mean square error of a point } & \multirow{2}{*}{$\mathbf{E}_{1}$} & \multirow{2}{*}{ 3D deviation } & \\
\hline & $m_{x}$ & $\boldsymbol{m}_{y}$ & $\boldsymbol{m}_{z}$ & $m_{P}$ & & & \\
\hline P1_1 & 0.000 & 0.000 & 0.000 & 0.000 & 0.000 & 0.000 & 0.017 \\
\hline P26_1 & 0.012 & 0.523 & 0.065 & 0.527 & 0.523 & 0.072 & 0.038 \\
\hline P51_1 & 0.017 & 1.353 & 0.091 & 1.356 & 1.390 & 0.182 & 0.048 \\
\hline P76_1 & 0.020 & 2.393 & 0.111 & 2.395 & 2.437 & 0.450 & 0.056 \\
\hline P101_1 & 0.023 & 3.609 & 0.128 & 3.611 & 3.658 & 0.562 & 0.063 \\
\hline P126_1 & 0.025 & 4.978 & 0.144 & 4.980 & 5.031 & 0.569 & 0.070 \\
\hline P151_1 & 0.028 & 6.547 & 0.158 & 6.549 & 6.541 & 0.950 & 0.076 \\
\hline P176_1 & 0.030 & 8.185 & 0.170 & 8.189 & 8.177 & 1.262 & 0.081 \\
\hline P201_1 & 0.032 & 9.939 & 0.183 & 9.941 & 9.930 & 3.090 & 0.087 \\
\hline
\end{tabular}


Table 2. Precision statistical results of classical free network.

\begin{tabular}{lllllll}
\hline$/ \mathbf{m m}$ & & & & & \\
\hline Precision index & Maximum of $\boldsymbol{m}_{\boldsymbol{P}}$ & RMS of $\boldsymbol{m}_{\boldsymbol{P}}$ & Maximum of $\mathbf{E}_{\mathbf{1}}$ & $\mathbf{R M S}_{\text {of }} \mathbf{E}_{\mathbf{1}}$ & $\mathbf{E}_{\mathbf{3}}$ & $\mathbf{E}_{\mathbf{4}}$ \\
\hline Value & 9.941 & 5.053 & 0.087 & 0.063 & 0.072 & 0.052 \\
\hline
\end{tabular}

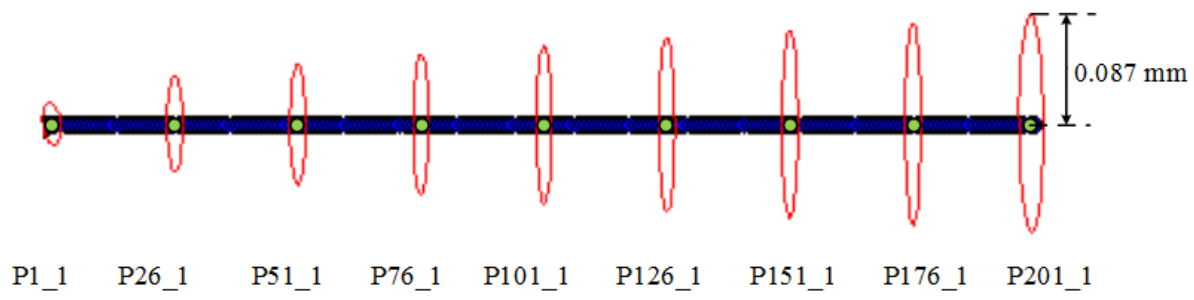

Figure 5. Distribution of relative error ellipses of classical free network.

It can be seen from Table 1 that the error accumulation is mainly in the $Y$-axis direction, which is caused by the low angle measurement accuracy of laser trackers. Over a long distance, the error accumulation caused by angle measurement error is very serious. Table 1, Figure 4 and Figure 5 show that the positional mean square error, the size of error ellipses, the $3 \mathrm{D}$ deviation and the size of relative error ellipses all increase with the distance from control points to the datum. At the end of the control network with a length of $1600 \mathrm{~m}$, the positional mean square error of $\mathrm{P} 201 \_1$ reaches $9.941 \mathrm{~mm}$, the length of semi-major axis of error ellipse of P201_1 reaches $9.930 \mathrm{~mm}$, and the $3 \mathrm{D}$ deviation is up to $3.090 \mathrm{~mm}$, which indicates that the positional precision and accuracy of the control points decreases gradually with the increase of the distance from the control point to the reference datum. It also demonstrates that taking the coordinate system of the first survey station as the global coordinate system and jointing multiple survey stations on a long distance will lead to serious error accumulation. In Table 1 and Figure 5, although the size of relative error ellipse of control points is small, it also increases gradually with the distance between control points and the datum, which indicates that relative positional position is also accumulated.

According to the above analysis, error accumulation refers to the phenomenon that the absolute positional precision and the relative positional precision gradually decrease with the increase of the distance from control points to the datum. The accumulation of absolute positional error is obvious, while the accumulation of relative positional error is not very serious. Particle accelerators mainly pursue high relative accuracy [26] and there is no specific requirement for absolute accuracy. However, in order to ensure the positional relationship between the main structures and facilitate segmental construction and installation, the absolute positional precision should be guaranteed as high as possible if conditions permit.

\section{Adjustment of Annexed Network}

An annexed network is a control network with redundant known conditions. The National Horizontal Control Network of China adopt the method of establishing networks from higher to lower orders [27]. They first build a nationwide primary control network with higher accuracy and lower density as a consistent control framework, and then continue to density the control network in a piecemeal fashion according to the needs of different areas. The side lengths of control networks become shorter and the accuracies get lower as the order changes from higher to lower. The lower level control network is forced to be attached to the higher level control network during adjustment calculation.

In order to provide global control, particle accelerators also need to establish a multi-layer control network [28, 29]. Generally, ground control network is constructed by GNSS observation or triangulation and trilateration, and then the coordinates of ground control points are projected and transferred into the tunnel. When the scale of the control network is very large, a tunnel backbone control network will be built to supply more intense control over a large span. The accuracy of National Horizontal Control Network is gradually reduced from higher to lower orders, while the accuracy requirements of ground control network, tunnel backbone control network and tunnel installation control network of particle accelerators are gradually increased. Therefore, the installation control network should not be compulsively attached to the ground control network or the tunnel backbone control network as the processing mode of the National Horizontal Control Network. It needs further study how to utilize the coordinates of ground control points and backbone control points to provide global control and reduce error accumulation.

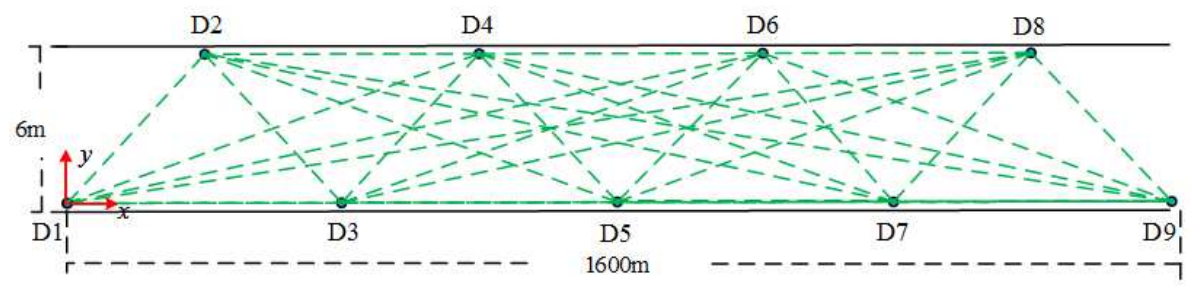

Figure 6. Tunnel backbone control network. 
The tunnel backbone control network of the $1.6 \mathrm{~km}$ linac is designed, as shown in Figure 6. It is arranged according to the straight stretch triangulation chain and consists of 9 control points from D1 to D9.

We take D1 as the origin of the coordinate system, the forward direction along the tunnel axis as the $x$ axis, and the upward direction of the plumb line on D1 as the $z$ axis, forming the right-hand coordinate system. The designed coordinates of backbone control points are shown in Table 3.

Table 3. Designed coordinates of backbone control points.

\begin{tabular}{llll}
\hline Point name & $\boldsymbol{x} / \mathbf{m}$ & $\boldsymbol{y} / \mathbf{m}$ & $\boldsymbol{z} / \mathbf{m}$ \\
\hline D1 & 0.000 & 0.000 & 0.000 \\
D2 & 200.000 & 6.000 & 0.000 \\
D3 & 400.000 & 0.000 & 0.000 \\
D4 & 600.000 & 6.000 & 0.000 \\
D5 & 800.000 & 0.000 & 0.000 \\
D6 & 1000.000 & 6.000 & 0.000 \\
D7 & 1200.000 & 0.000 & 0.000 \\
D8 & 1400.000 & 6.000 & 0.000 \\
D9 & 1600.000 & 0.000 & 0.000 \\
\hline
\end{tabular}

The nominal angle measurement accuracy of Leica TS60 total station is $\pm 0.5^{\prime \prime}$, and its nominal ranging accuracy is \pm $\left(0.6 \mathrm{~mm}+1 \times 10^{-6} \times \mathrm{D}\right)$. According to the nominal accuracy, simulated observations of tunnel backbone control network is conducted with method in all combinations. Assuming that D1 and D9 are two projected points from the GNSS network on the ground, and their horizontal coordinate accuracy is $\pm 3 \mathrm{~mm}$. The leveling between D1 and D9 is simulated according to the specifications for the second-class leveling.
It is known that the distance from D1 to D9 is $1.6 \mathrm{~km}$, so the leveling route is about $3.2 \mathrm{~km}$. The total mean square error MW of per kilometer of the second-class leveling is $2.0 \mathrm{~mm}$, so we can calculate that the elevation mean square error of the weakest point is $\mathrm{M}_{\mathrm{II}}=\mathrm{M}_{W} \times \sqrt{S / 4}=2.0 \mathrm{~mm}$. Then, we add 3 $\mathrm{mm}$ to the horizontal coordinate and $2 \mathrm{~mm}$ to the elevation coordinate of D9, and take D1 and D9 as datums. The adjusted coordinates and precision of each point are shown in Table 4.

Table 4. Adjusted coordinates and precision of backbone control points.

\begin{tabular}{lllllll}
\hline $\begin{array}{l}\text { Point } \\
\text { name }\end{array}$ & $\mathbf{x} / \mathbf{m}$ & $\mathbf{y} / \mathbf{m}$ & $\mathbf{z} / \mathbf{m}$ & $\boldsymbol{m}_{\mathbf{x}} / \mathbf{m m}$ & $\boldsymbol{m}_{\mathbf{y}} / \mathbf{m m}$ & $\boldsymbol{m}_{\mathbf{z}} / \mathbf{m m}$ \\
\hline D1 & 0.0000 & 0.0000 & 0.0000 & 0.000 & 0.000 & 0.0 \\
D2 & 200.0013 & 6.0003 & 0.0002 & 0.332 & 0.308 & 1.0 \\
D3 & 400.0013 & 0.0008 & 0.0005 & 0.331 & 0.359 & 1.0 \\
D4 & 600.0014 & 6.0011 & 0.0008 & 0.330 & 0.381 & 1.4 \\
D5 & 800.0015 & 0.0016 & 0.0010 & 0.329 & 0.388 & 1.4 \\
D6 & 1000.0017 & 6.0019 & 0.0013 & 0.330 & 0.381 & 1.4 \\
D7 & 1200.0021 & 0.0023 & 0.0015 & 0.331 & 0.359 & 1.7 \\
D8 & 1400.0019 & 6.0026 & 0.0018 & 0.332 & 0.308 & 1.7 \\
D9 & 1600.0030 & 0.0030 & 0.0020 & 0.000 & 0.000 & 2.0 \\
\hline
\end{tabular}

Taking the adjusted coordinates of the 9 tunnel backbone control points in Table 3 as mandatory datums, the coordinate adjustment of the installation control points is carried out. In this adjustment, the installation-control- network is compulsively attached to the backbone control network. The distribution of error ellipses is shown in Figure 7, and the distribution of relative error ellipses between two adjacent control points is shown in Figure 8.

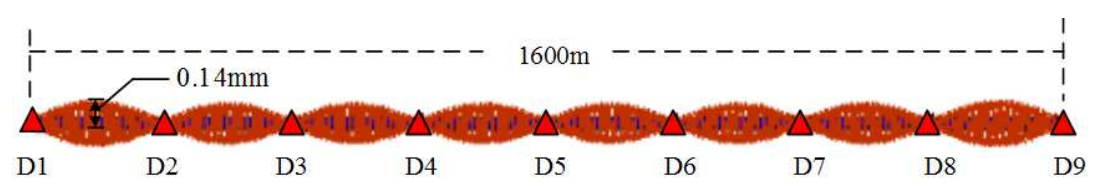

Figure 7. Distribution of error ellipses of annexed network.

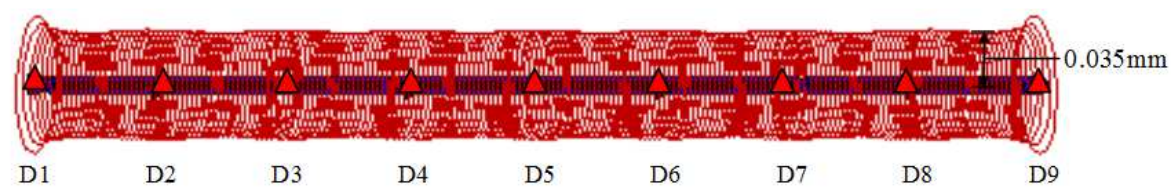

Figure 8. Distribution of relative error ellipses of annexed network.

The distribution of error ellipses in Figure 7 has regularity. The installation control network is divided into 8 segments with the 9 backbone control points, and the distribution of error ellipses on each segment is almost the same. The size of the error ellipses are small which distribute near to the backbone control points, and the size of the error ellipses are large which distribute at the middle of two backbone control points. The length of the semi-major axis of the largest error ellipse is $0.14 \mathrm{~mm}$. The comparison between Figure 7 with Figure 4 reveals that the accumulation of absolute positional precision is effectively reduced by taking the backbone control points as mandatory datums. Figure 8 shows that, except for the relative error ellipses near to D1 and D9, the size of most relative error ellipses is approximately the same, which indicates that the accumulation of relative positional precision is also reduced by taking the backbone control points as the datum.

In Figure 7, the distribution of error ellipses is almost the same on the 8 segments, so we take the D1 D2 segment as an example and select 6 control points at an interval of $40 \mathrm{~m}$. The precision of the 6 points are listed in Table 5. The precision statistical results of annexed network adjustment are shown in Table 6. 
Table 5. Precision of some control points in annexed network.

\begin{tabular}{|c|c|c|c|c|c|c|c|}
\hline \multicolumn{8}{|l|}{$/ \mathrm{mm}$} \\
\hline \multirow{2}{*}{ Point name } & \multicolumn{4}{|c|}{ Mean square error of a point } & \multirow{2}{*}{$\mathbf{E}_{1}$} & \multirow{2}{*}{ 3D deviation } & \multirow{2}{*}{$\mathbf{E}_{2}$} \\
\hline & $m_{x}$ & $\boldsymbol{m}_{y}$ & $\boldsymbol{m}_{z}$ & $m_{P}$ & & & \\
\hline P1_1 & 0.000 & 0.000 & 0.000 & 0.000 & 0.000 & 0.000 & 0.044 \\
\hline P6_1 & 0.012 & 0.085 & 0.057 & 0.103 & 0.098 & 2.119 & 0.036 \\
\hline P11_1 & 0.012 & 0.136 & 0.063 & 0.150 & 0.136 & 1.906 & 0.034 \\
\hline P16_1 & 0.013 & 0.128 & 0.063 & 0.143 & 0.128 & 1.222 & 0.034 \\
\hline P21_1 & 0.013 & 0.081 & 0.056 & 0.099 & 0.081 & 1.113 & 0.036 \\
\hline P26_1 & 0.013 & 0.028 & 0.046 & 0.055 & 0.028 & 1.111 & 0.035 \\
\hline
\end{tabular}

Table 6. Precision statistical results of annexed network.

\begin{tabular}{lllllll}
\hline$/ \mathbf{m m}$ & & & & \\
\hline Precision index & Maximum of $\boldsymbol{m}_{\boldsymbol{P}}$ & RMS of $\boldsymbol{m}_{\boldsymbol{P}}$ & Maximum of $\mathbf{E}_{\boldsymbol{1}}$ & ${\text { RMS of } \mathbf{E}_{\boldsymbol{1}}}_{\mathbf{E}_{3}}$ & $\mathbf{E}_{\mathbf{4}}$ \\
\hline Value & 0.153 & 0.110 & 0.044 & 0.035 & 0.478 & 0.094 \\
\hline
\end{tabular}

It can be seen from Table 6 and Table 2 that the RMS of $m_{\mathrm{P}}$ decreased significantly by taking the backbone control points as mandatory datums. The maximum of $m_{\mathrm{P}}$ is only $0.153 \mathrm{~mm}$, indicating that the accumulation of absolute positional error is well controlled. The maximum and RMS of semi-major axis of relative error ellipses of annexed network is also smaller than that of classical free network, and the RMS of semi-major axis of relative error ellipses of annexed network is only 0.035 $\mathrm{mm}$, which indicates that the annexed network also effectively inhibits the accumulation of relative positional error. However, when comparing the size of relative error ellipses distributing on the P1_1 P26_1 segment, it can be found that the length of semi-major axis of relative error ellipses of classical free network varies almost linear from $0.017 \mathrm{~mm}$ to $0.038 \mathrm{~mm}$, while the length of semi-major axis of relative error ellipses of annexed network are all about $0.035 \mathrm{~mm}$, which indicates that it may decrease the relative precision between some control points to compulsively attach the installation control network to the backbone control network. Let us make an analysis for the reasons. In the annexed network adjustment, we take the backbone control points as mandatory datums, so the coordinates of backbone control points have no change before and after adjustment. The internal geometry of installation control network is compulsively jointed with the mandatory datums, which will lead to changes in the shape and scale of the control network. What's more, the precision of the backbone control points is not very high, and the inconsistencies between the adjustment coordinates and the true coordinates will be all allotted to the installation control network, which results in the loss of relative precision between some control points.

For particle accelerators, the relative precision between control points is the primary precision requirement, while the absolute precision of control points is the secondary precision requirement. It is unreasonable to accept the data processing scheme which improves the absolute precision while reduces the relative precision. Therefore, it is necessary to study a new method for the data processing of installation control network and backbone control network.

\section{Sectional Control Method Combining Mandatory Datum and Faint Datum}

In order to keep the relative precision as high as possible and avoid big absolute error at the same time, this paper puts forward a new method. It can be seen from Figure 3 that the positional mean square error and the size of error ellipses of control points increase with the distance from control points to the datum. Therefore, in order to decrease the error accumulation, the distance from control points to the datum should be confined. We can confine the distance by dividing control network into several subsections, and appoint appropriate datum on each segment, so as to ensure that the distance from each control point to the datum is no more than a certain value.

The length control network segment should be determined by comprehensively considering the positional precision of backbone control points and the value of error accumulation of installation control network. The segmentation process needs to follow the basic principle that each segment should be as long as possible in order to reduce the number of segments, while the length of segment must not exceed a certain value to confine the error accumulation. As shown in Table 1, error accumulation is mainly caused by the accumulation of horizontal positional error. On the other hand, and the elevation precision is affected by complex factors such as the earth gravity field, the selection of height datum, and so on. Therefore, we only take the horizontal positional precision as the threshold. When the value of error accumulation exceeds the horizontal positional precision of the backbone control network, the installation control network should be segmented reasonably. The horizontal positional precision of the backbone control network of the linac is about $0.5 \mathrm{~mm}$, and the mean square error of the control point is $0.53 \mathrm{~mm}$ which is $200 \mathrm{~m}$ away from the datum. So we can select $200 \mathrm{~m}$ as the threshold. By appointing the middle survey station as the datum, each segment can cover a range of $400 \mathrm{~m}$, and the $1.6 \mathrm{~km}$ long control network can be divided into 4 subsections. After segmentation, adjustment is carried out independently 
in the local coordinate system on each segment. The distribution of error ellipses of the installation control network is shown in Figure 9, and the distribution of relative error ellipses is shown in Figure 10.

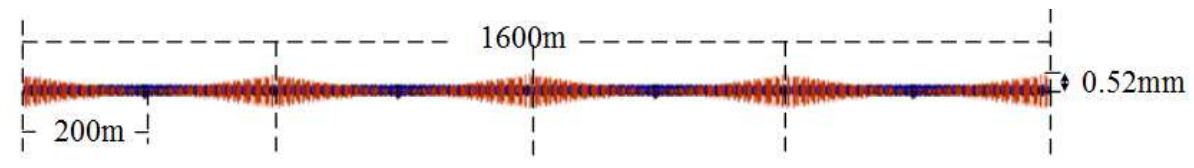

Figure 9. Distribution of error ellipses after subsection.

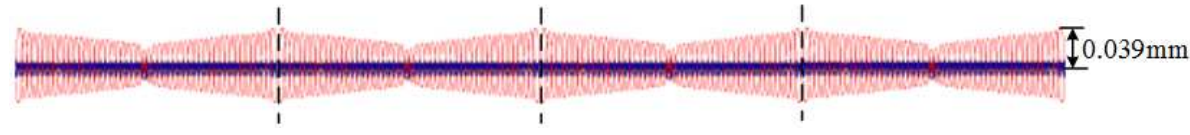

Figure 10. Distribution of relative error ellipses after subsection.

Through comparison of Figure 9 and Figure 4, we find that the size of error ellipses decreased obviously after segmentation, and the length of semi-major axis of the largest error ellipse is only $0.520 \mathrm{~mm}$, indicating that the accumulation of absolute positional precision is effectively controlled.

Although the error accumulation can be decreased with segmentation, the control points only have the coordinates in the independent coordinate system on each segment, which cannot provide reference information in the unified global coordinate system. The coordinate systems on each segment should be unified and transformed to a global coordinate system. The coordinates of control points on the 4 segments in separate coordinate systems and the coordinates of the 9 backbone control points are regarded as independent observed values. By taking the coordinate system of backbone control network as the unified global coordinate system, the weighted partial parameters adjustment is carried out. In this adjustment, the coordinates of the backbone control points are given small weight, and the given weight in this example is 0.5 . We name the above data processing method as segmental control method. Its specific steps are as follows.

Step 1: Carry out simulation calculation to get the variation rule of positional mean square error with the distance from control point to the datum according to the shape of the tunnel installation control network.

Step 2: Take the horizontal positional precision of the backbone control network as the threshold. When the value of error accumulation exceeds the threshold, the installation control network should be segmented reasonably.

Step 3: Appoint the middle survey station on each segment as mandatory datum, and carry out the adjustment calculation independently in the local coordinate system on each segment.

Step 4: Take the coordinate system of backbone control network as the unified global coordinate system. Give a small weight to the coordinates of backbone control points and perform weighted partial parameters adjustment by taking the adjusted coordinates on control points on each segment and the coordinates of backbone control points as independent observations.

After segmentation, the distribution of error ellipses and relative error ellipses (Figure 9 and Figure 10) on each segment is almost the same, and the distribution trend on each segment is symmetric about the middle survey station. Therefore, we take the $0 \sim 200 \mathrm{~m}$ segment as an example and selected 6 points at an interval of $40 \mathrm{~m}$. The precision of the 6 points of sectional control method is shown in Table 7. The precision statistical results of sectional control method are shown in Table 8 . The variation of the difference of positional deviation between adjacent points with point number is shown in Figure 11.

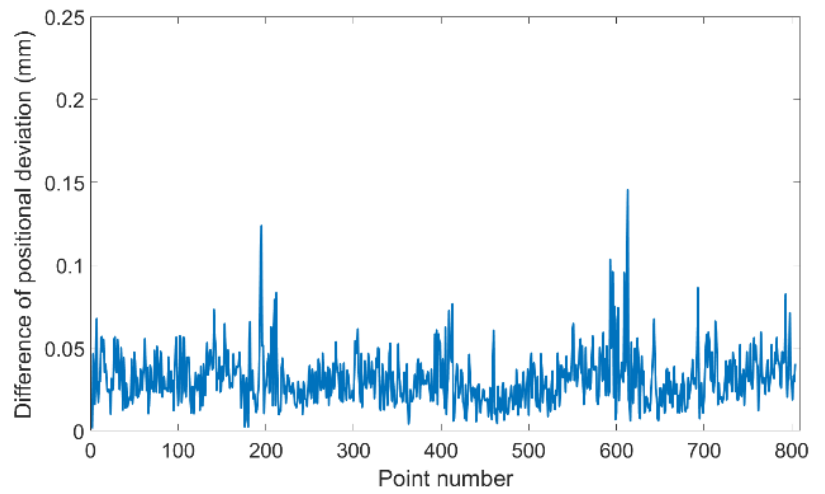

Figure 11. Variation of difference of positional deviation with point number.

Table 7. Precision of some control points of sectional control method.

\begin{tabular}{|c|c|c|c|c|c|c|c|}
\hline \multirow{3}{*}{ Point name } & & & & & & \multirow{3}{*}{ 3D deviation } & \multirow{3}{*}{$\mathbf{E}_{2}$} \\
\hline & \multicolumn{4}{|c|}{ Mean square error of a point } & \multirow{2}{*}{$\mathbf{E}_{1}$} & & \\
\hline & $m_{x}$ & $m_{y}$ & $\boldsymbol{m}_{z}$ & $m_{P}$ & & & \\
\hline P1_1 & 0.014 & 0.453 & 0.069 & 0.458 & 0.453 & 0.122 & 0.040 \\
\hline P6_1 & 0.011 & 0.330 & 0.059 & 0.335 & 0.329 & 0.104 & 0.033 \\
\hline P11 1 & 0.010 & 0.220 & 0.052 & 0.226 & 0.220 & 0.082 & 0.031 \\
\hline P16_1 & 0.008 & 0.126 & 0.043 & 0.133 & 0.126 & 0.081 & 0.027 \\
\hline P21_1 & 0.007 & 0.052 & 0.033 & 0.062 & 0.052 & 0.032 & 0.024 \\
\hline P26_1 & 0.000 & 0.000 & 0.000 & 0.000 & 0.000 & 0.000 & 0.015 \\
\hline
\end{tabular}


Table 8. Precision statistical results of sectional control method.

\begin{tabular}{lllllll}
\hline$/ \mathbf{m m}$ & & & & \\
\hline Precision index & Maximum of $\boldsymbol{m}_{\boldsymbol{P}}$ & RMS of $\boldsymbol{m}_{\boldsymbol{P}}$ & Maximum of $\mathbf{E}_{\mathbf{1}}$ & RMS of $\mathbf{E}_{\mathbf{1}}$ & $\mathbf{E}_{\mathbf{3}}$ & $\mathbf{E}_{\mathbf{4}}$ \\
\hline Value & 0.546 & 0.260 & 0.039 & 0.029 & 0.145 & 0.035 \\
\hline
\end{tabular}

By comparing Table 8, Table 6 and Table 2, it can be seen that the RMS of positional mean square error of sectional control method is $0.260 \mathrm{~mm}$, which is less than the result of classical free network but greater than that of annexed network. The value of this precision index reflects that sectional control method decreases the accumulation of positional error to a certain extent. Although its control effect is not as good as that of annexed network, the maximum of $m_{P}$ is only $0.546 \mathrm{~mm}$, which indicates that the accumulation of positional error is well controlled and the result is acceptable. The RMS of semi-major axis of relative error ellipses of annexed network is $0.035 \mathrm{~mm}$, while that of sectional control method is $0.029 \mathrm{~mm}$. The RMS of the difference of positional deviation of annexed network between adjacent points is $0.094 \mathrm{~mm}$, while that of sectional control method is $0.035 \mathrm{~mm}$.
The above comparison indicates that the relative positional precision of sectional control method is superior to the relative positional precision of annexed network. Figure 11 shows the variation of point position deviation with point number. It can be seen that, except for a few points which are adjacent to the backbone control points, the difference of positional deviation of most control points is around $0.05 \mathrm{~mm}$, which shows that sectional control method effectively guarantees the relative positional precision. To sum up, the sectional control method has achieved significant results in decreasing error accumulation. It is mainly due to the reasonable selection of datums. From the perspective of the entire data processing progress, the distribution of datums which are adopted by sectional control method is shown in Figure 12.

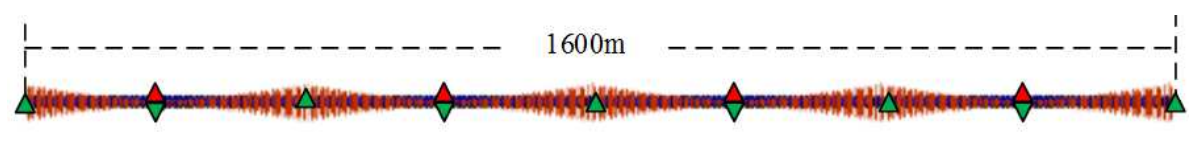

$\Delta$ Faint datum $\Delta$ Mandatory datum

Figure 12. Distribution of datums.

Figure 12 illustrates that the essence of sectional control method is to joint and unify all the survey stations by combining mandatory datums and faint datums. Firstly, the middle survey station on each segment is appointed as mandatory datum to ensure that the positional precision of control points will not exceed a certain value. Then, the backbone control points are taken as faint datums, which can provide the unified global coordinate reference. Finally, the weighted partial parameters adjustment is conducted, in which the backbone control points are given small weight to avoid the destruction of the relative precision of adjacent control points.

\section{Conclusion}

The problem of error accumulation in the tunnel installation control network of particle accelerator is studied in this paper, and a sectional control method is proposed to decrease the error accumulation. The effect of the proposed method is confirmed by the simulation measurement data of a linac control network. The main conclusions are as follows.

(1) Error accumulation mainly refers to the phenomenon that the absolute positional precision and the relative positional precision gradually decrease with the increase of the distance from the control point to the datum. The accumulation of absolute positional error is obvious, while the accumulation of relative positional error is not very serious. The error accumulation is very serious over long distance, and cannot be ignored for large particle accelerators.

(2) The annexed network takes the backbone control points as mandatory datums, which compulsively attaches installation control network to backbone control network. Due to that the precision of the backbone control points is not very high, the inconsistencies between the adjustment coordinates and the true coordinates of backbone points will be all allotted to the installation control network, which will results in the loss of relative precision between some control points.

(3) The proposed sectional control method unifies the coordinate systems of all survey stations while considering the relative and the absolute precision. It combines mandatory datums and faint datums to process the observation data of the tunnel installation control network, which not only ensures the relative precision of the control points, but also decreases the error accumulation.

(4) The downside is that the weight of backbone control points is not easy to be determined. After many tests and calculations, we found that the shape of installation control network will be distorted to a certain extent when the weight of backbone control points is too large, which will affect the relative precision of adjacent control points, and the backbone control points will lose its inhibitory effect on error accumulation when the weight is too small. Therefore, how to determine the value of the weight needs to be further studied. Furthermore, how to construct the control network efficiently and accurately also need to be further studied. 


\section{References}

[1] Raghavan J. (2012). Particle Accelerators, Colliders, and the Story of High Energy Physics: Charming the Cosmic Snake (Springer, Berlin Heidelberg), pp. 41-66.

[2] Chen H. S., Wang X. L. (2016) China's first pulsed neutron source. Nat. Mater. 15 (7), 689-691.

[3] Wei J. (2016). Particle accelerator development: Selected examples. Mod. Phys. Lett. A, 31 (10), 1630010.

[4] Kume, T., Satoh, M., Suwada T., et al. (2013). Large-scale accelerator alignment using an inclinometer. Precis Eng. 37 (4), 825-830.

[5] Li G. Y., Fan B. X. (2017). The development of precise engineering surveying technology. Acta Geod. et Cartogr. Sinica. 46 (10), 1742-1751.

[6] Silva, T. Martins, M. N. (2011). Statistical treatment of misalignments in particle accelerators. Comput. Phys. Commun. 182 (3). 679-682.

[7] Guo Y. G. Li Z. C. Li G. Y. et al. (2020). Progress and Prospect of Engineering Control Network for Particle Accelerator. Bull. Surv. Mapp. 2020 (1), 136-141.

[8] Cai G. Z., Man K. D., Wang S. M. et al. (2013). A Combined Application of Laser Tracker and Spatialanalyzer in Alignment of Acelerator. Applied Mechanics and Materials. 333-335.

[9] Guo Y. G., Li Z. C., Zhao W. B., et al. (2020). Two-laser-tracker system for precise coordinates transmission. Opt. Precis. Eng. 28 (1), 30-38.

[10] O. Bezditko, A. Zelinsky, I. Karnaukhov, et al. (2017). NSC KIPT experience in use of laser tracker Leica at 401 in equipment alignment of $100 \mathrm{mev} / 100 \mathrm{kw}$ electron linear accelerator of "Neutron Source" driver. in Proceedings of IPAC2017, Copenhagen, Denmark, THPVA069, pp. 4604-4606.

[11] Yuan J. D., He Y., Zhang B., et al. (2017). Alignment of beam position monitors in cryomodule of CADS injector II. Nucl. Sci. Tech. 28, 75.

[12] Liang J., Dong L., Luo T., et al. (2013). Precision statistics of laser tracker in BEPCII storage ring and calculation of mean square error of unit weight. Sci. Surv. Mapp. 38 (6), 182-184.

[13] Yu C. H., Ke M., Du H. W. et al. (2006). Application Research of 3D Control Network Technology in BEPC II Storage Ring. Chin. Phys. C 30 (11), 1107-1112.

[14] Yang F. (2014). Theories and methods of control network on accelerator (The PLA information engineering university, Zhengzhou).

[15] Jiang X. M., Wang J. Q., Qin Q. et al. (2014). Chinese high energy photon source and the test facility. SCIRNTIA. SINICA. Phys., Mech. \& Astron., 44 (10), 1075-1094.

[16] Wang Y. F. (2018) From BEPC to CEPC. Mod. Phys. 30 (5), 62-66.

[17] CEPC Conceptual Design Report. (2018). (Institute of High Energy http://cepc.ihep.ac.cn/CEPC_CDR_Vol1_Accelerator. Physics,)

[18] Dominique. M. (2018). The Survey and Alignment of the FCC Paper presented at the 4th FCC Week, Beurs van Berlage, Amsterdam, 9-13 April.

[19] Zhou Y. Y., Pan G. R., Wu T., et al. (2017). Influence on Selection of Data Fusion Model to Overall Adjustment in Industrial Measurement. Geomat. Inf. Sci. Wuhan Univ. 42 (12), 1840-1846.

[20] Lü D., Liu S. G., Fan C. Y., et al. (2018). Overall Adjustment Method of Coordinate Transforming Measurement Data In Global Measurement Space. Sci. Surv. Mapp. 12 (43), 137-144.

[21] Sui L. F., XU Q. F., (2002). Reference Stations Weight Attached and Its Influence in GPS Data Processing. J. Inst. of Surv. Mapp. 19 (4), 235-242.

[22] Tao B. Z. (2001). Free network adjustment and deformation analysis (Surveying and Mapping Press, Beijing), pp. 35-48.

[23] Sui L. F., Song L. J., Chai H. Z., et al. (2016). Error Theory and Foundation of Surveying Adjustment, 2nd edn. (Surveying and Mapping Press, Beijing), pp. 205-208.

[24] Zhou J. W., Tao B. Z., Zhuang K. Y. (1987). Proceedings of Quasi-Steady adjustment (Surveying and Mapping Press, Beijing), pp. 17-25.

[25] Yang Y. X., Zhang L. P., (2007). Maintenance of Coordinate Datum and Data Processing of Dynamic Monitoring Network. Geomat. Inf. Sci. Wuhan Univ. 32 (11), 967-971.

[26] Wang W. (2016).Survey and alignment of the HLS II upgrade project and study of the measurement precision (University of Science and Technology of China, Hefei).

[27] Lü Z. P., Qu Y. Y., Qiao S. B., (2014). Geodesy: Introduction to Geodetic Datum and Geodetic Systems (Springer, Berlin Heidelberg), pp. 76-77

[28] Gervaise J. (1985). CERN Accelerator School applied geodesy for particle accelerators. J Geod. 59, IX-X.

[29] Ruland, R. E. (1994). Survey and Alignment of Particle Accelerators and Transport Lines. Jour. Surv. Eng. 120 (1), 11-24. 